\title{
Giant Atypical Lipomatous Tumor of Posterior Mediastinum In Adolescent \A Case Study and the Literature Review
}

\section{Yang Zhang}

First Affiliated Hospital of Guangdong Pharmaceutical College

\section{Kai Chen}

First Affiliated Hospital of Guangdong Pharmaceutical College

Qing-Rong Ma

First Affiliated Hospital of Guangdong Pharmaceutical College

\section{Zhuang-Rong Huang}

First Affiliated Hospital of Guangdong Pharmaceutical College

Shu-Guang Zhu ( $D$ zhusg65@126.com )

First Affiliated Hospital of Guangdong Pharmaceutical College

\section{Case report}

Keywords: Liposarcoma, Mediastinal neoplasms, well-diferentiated liposarcoma/atypical lipomatous tumor, Molecular inhibitors

Posted Date: December 21st, 2020

DOI: https://doi.org/10.21203/rs.3.rs-131033/v1

License: (c) (i) This work is licensed under a Creative Commons Attribution 4.0 International License. Read Full License 


\section{Abstract}

Background: It is usually common for liposarcoma as one of the soft tissue sarcomas to be found in adults, and the disease in children is the rare case.

Case presentation: This article involves in a 14-year-old boy with a primary posterior mediastinal atypical lipomatous tumor. It also provides a literature review and summary for the disease with the aim of making the disease well learned, because of the absence of the uniform standards for the clinical features, diagnostic methods, best treatment methods, and clinical efficacy of the atypical lipomatous tumor.

Conclusion: Complete surgical resection is the best method for the eradication of tumors. However, mediastinal liposarcoma can not be completely removed by surgery in some cases. For the treatment of residual tumor tissue, molecular inhibitors will be an important supplementary method for the treatment of liposarcoma in the future.

\section{Introduction}

Liposarcoma is a type of malignant adipocytic tumor. According to NCCN (National Comprehensive Cancer Network) in 2019, there are four classifications for liposarcoma: Well-differentiated, myxoid, pleomorphic, and dedifferentiated. Among them the most common one is well-differentiated liposarcoma/Atypical lipomatous tumor(WDL/ALT), which mainly occurs in the extremities and retroperitoneum and is rarely seen in the mediastinum [1]. The exact proportion of liposarcoma remains unknown, and the misdiagnosis rate for it is high. By far, there are limited cases of mediastinal atypical lipomatous tumor been reported; therefore, its clinical characteristics, optimal treatment, diagnosis methods and clinical outcomes remain unclear.

This study documents a 14-year-old male patient with a primary posterior mediastinal atypical lipomatous tumor, and provides a retrospective analysis of the clinical features, treatment and prognosis of the well-differentiated liposarcoma through the learning of Pubmed 1949-2020 related cases.

\section{Case Report}

A 14-year-old boy was hospitalized after " two-week cough with sputum and five-day pain in left chest". CT (computed tomography) from other hospital showed that there was a large mass on the left thorax with dense shadows and the left lung was poorly inflated. There are no past medical history, family history, or any relevant genetic information about the disease in this patient.

The comprehensive examination results of admission showed CA-125 reaching $44.2 \mathrm{U} / \mathrm{ml}$; and CT result showed a large irregularity and density in the left mediastinum and thoracic cavity (Fig. 1A), with the margin being locally unclear and the obvious low density $(-84 \mathrm{Hu})$ and high density $(24 \mathrm{HU})$ areas in the mass. After the enhancement, neither obvious enhancement in any of the stages nor obviously enlarged 
lymph nodes were observed. After contrast-enhanced, the unevenness of mass was enhanced; the adjacent left lung tissue was locally compressed and collapsed; and the mediastinum and trachea shifted to the right. The source of the tumor could not be clarified through the film (Fig. 1B). A huge fatcontaining tumor lesion in the left mediastinum-left thoracic cavity was identified after imaging examination was conducted. And then an immediate left thoracentesis biopsy was given to the patient. It is can only be confirmed as fatty tumors according to the pathological results. No abnormality was found in cranial MR (magnetic resonance), cardiac color Doppler ultrasound, organ color Doppler ultrasound. Considering that the benefits of chest MR are quite limited in identifying the source of the patient's tumor, the patient chose surgery to relieve the persistent chest pain and cough symptoms. During the operation, the tumor was inserted into the chest through the fifth intercostal level along the posterior side of the left chest. The inspection showed that, almost occupied the thoracic cavity, the tumor has a complete capsule, which has a size of about $20^{\star} 15^{\star} 11 \mathrm{~cm}$ (Fig. 1C, D), and closely adheres to the chest wall and mediastinum. The mass originates from the upper left posterior chest wall and its relationship with with the mediastinal vessels remains unknown. After completely resecting and removing the tumor, we found a little bleeding in the lung tissue of the upper left lung to which the tumor adhered, and therefore suspected the existence of tumor tissue. After wedge resection, the tumor was sent for pathological examination. After the completion of the operation seven days later when the indwelling thoracic drainage tube was removed from the patient, the re-examination of the chest CT showed a good left lung recruitment (Fig. 1E), and no obvious soft tissue mass was found.

\section{Final diagnosis}

Inside the tumor, it can be seen from the cut section that the adipose tissue with blood vessels running and a partial myxoid appearance(Fig. 2A,B,C). Through the hematoxylin and eosin staining, it can be found the tumor tissue is composed of fat vacuoles of varying sizes, branched capillaries, short spindleshaped fibroblast-like cells and immature adipocytes. Among them what can be observed is adipocytes, with its quality having the obvious myxoid appearance (Fig. 3A,B). Immunohistochemical analysis shows that the tumor is positive for $\mathrm{S} 100$ (S100 Proteins) and CD34(Antigens,CD34), and the ki67(ki-67 Antigen) index is 5\%. Genetic test shows negative results for MDM2 (Murine Double Minute2) and CHOP (DDIT3/DNA Damage Inducible Transcript 3). Finally, we established the diagnosis of atypical lipomatous tumor with mucinous change secondary myxoid changes in the left posterior mediastinum.

\section{Outcome and Follow-up Study}

The patient made a full recovery after the operation. The genetic test results show that the MDM2 and $\mathrm{CHOP}$ genes are negative, so the lesion is considered to be a low-grade tumor. Given the young age of the patient and the unproved effectiveness of the adjuvant therapy, the child was discharged from the hospital after his condition becomes stable. In the following five months no signs of recurrence are found in the patient through the observation. The contact with the patient's family will continue in the future to come. 


\section{Discussion}

WDL/ALT mostly occurs in the elderly over 60 years old and its occurrence in adolescents is occasional[2]. There the incidence among male and female shows no significant difference. This kind of tumors is common in the lower extremities and the retroperitoneum, but its origin in the mediastinum is extremely rare. Most liposarcomas show themselves as large, painless masses, and the finding of them are usually the result of symptoms caused by some kinds of trauma or compression of important organs. The common symptoms of mediastinal liposarcoma include chest pain, dyspnea, cough, and weight loss. They mostly occur in the anterior mediastinum and often involve the pericardium, diaphragm, and phrenic nerve[3, 4]

In this article, we make a literature review by obtaining the English and Japanese literatures with available patient case information between 1949 to 2020 from PubMed. Keywords we used are Liposarcoma, mediastinum or mediastinal, and, as a result, 61 cases of primary mediastinal liposarcoma including the one detailed in this study are identified. Most of these cases occurred in adults, and six reported were in children. The mean patient age is 53 years old, with an age range of 5 to 81 and a predilection for males (41 males and 20 females). The pathological subtype is that well-differentiated liposarcoma has 25 cases (41\%), myxoid liposarcoma 16 cases (26\%), dedifferentiated liposarcoma 10 cases (16\%), pleomorphic liposarcoma four cases (7\%), mixed liposarcoma five cases (8\%) and recurrent liposarcoma one cases (2\%). There were 36 cases with detailed symptom descriptions, 36 with some symptoms and 25 without symptoms. The common complaints include dyspnea (18 cases), chest pain (five cases), and cough (five cases); and the other symptoms are weight loss, hoarseness, superior vena cava syndrome manifestations, fever, arrhythmia, and dizziness. The position where the tumor most frequently occurred is the anterior ( 26 cases respectively), followed by the posterior ( 16 cases) and the anterior-middle mediastinum (one case). The size of the tumor size varies, from $1 \mathrm{~cm}$ to $40 \mathrm{~cm}$, and the mean size is $18.9 \mathrm{~cm}$. There are only seven cases with the tumor size below $10 \mathrm{~cm}$ and 17 cases with the tumor size above $20 \mathrm{~cm}$ in diameter. Besides, 37 cases are those failing to obtain tumor size information. There are only five reporting tumor invasion of surrounding structures, including pleura, esophagus, pericardium and phrenic nerve. There is only one case of distant metastasis, with lung being the metastasis site.

In terms of initial management, 53 patients receive the complete surgical resection, two receive the incomplete surgical resection, one receives chemotherapy and five are reported unknown. Of the 55 surgical cases 10 patients receive adjuvant therapy after surgery (three for chemotherapy, four for radiotherapy and three for both). Unfortunately, the recurrence of the tumor is found in nine cases $(9 / 55$, $16.4 \%$ ) after surgery. Of the 61 total patients 38 cases survive without evidence of disease, seven cases die (five cases dead of disease and two cases dead of other diseases), and 15 cases are missing. The longest survival time is 114 months.

From related literature reports, we can find a consistent conclusion that the complete surgical resection is the best method for the eradication of tumors.Yan[5] pointed out that patients with tumor located under the skin can be cured after complete resection of the tumor, while those with tumor located in the deep 
part are prone to recurrence because the tumor cannot be completely removed. Therefore, Fukuhara $\mathrm{S}$ et al.[6] proposed that the surgical procedures for liposarcoma can be intralesional (within the tumor mass, often leaving gross tumor), marginal (through the surrounding fibrous membrane, often leaving microscopic foci of tumor), wide (outside the membrane and compartment, leaving no tumor other than "skip metastases"), and radical (most often involving the entire limb and including the entire compartment in which the tumor was located). Since the posterior mediastinal liposarcoma tissue is usually adjacent to important organs such as the heart, aorta, lung, superior vena cava, phrenic nerve, and diaphragm, the marginal surgery can be the target of surgical intervention, which is more reasonable. When the complete removement of the tumor failed, the non-radical resection will be an unfavorable factor affecting overall survival [7]. In the report, Derbel et al. [8] pointed out that the median time from diagnosis to first recurrence of soft tissue sarcoma is 11.5 months, and the recurrence rate of mediastinal liposarcoma is as high as $40 \%$. Therefore, at least one-year follow-up is required for patients with this type of tumor after operation.

In addition, the location and type of the tumor are identified as two important factors which affect the prognosis of liposarcoma. According to results of most studies until now, the incidence of mediastinal liposarcoma is seemingly often found in the anterior mediastinum. With the rarity of case of the posterior mediastinal liposarcoma, there still might be a close interrelationship between its tumor behavior and the low mortality, recurrence rate and metastasis rate [9]. However, since tumors located in the central cavity tend to wrap around important structures, or they can simulate normal adipose tissue well, it is difficult to achieve the ideal goal by the marginal surgery. The most common histological types of mediastinal liposarcomas are well-differentiated and myxoid. All types of liposarcomas contain different numbers and morphologies of diploblasts. However, the less differentiated liposarcomas show more aggressive and local and distant complications. As mentioned above, liposarcomas grow in a more insidiously manner, with usually larger size. The finding of masses is accidental due to the compression or stimulation of adjacent structures. Most related symptoms can be relieved after tumor resection, and the survival time is not affected. Therefore, the tumor size has no influence on the prognosis.

No definite diagnosis can be made through the tissue biopsy. ALT/WDL is composed of mature adipocytes with deep stained nuclei without any solid areas. However, a complete sampling of the tumor is required for atypical mesenchymal cells to help clarify. Although the existence of tumor tissue can be confirmed from the results of tissue biopsy in this case, we are more inclined to the diagnosis of myxoid liposarcoma. In order to distinguish benign and malignant, we further improved MDM2 and CHOP gene detection. It can be seen that the tissue biopsy only serves as a preliminary screening, and the fluorescence in situ hybridization (FISH) method can provide more accurate results. Interestingly, MDM2 and $\mathrm{CHOP}$ genes are negative in this case. Several experienced pathologists identified that there is still a similarity between the nature of the tumor that of adult well-differentiated liposarcoma. Finally, the diagnosis of this case is confirmed as low-grade malignant liposarcoma.

Saeed et al. [10] performed the surgery on a 17-year-old girl with pleomorphic liposarcoma of the anterior mediastinum, followed by adjuvant chemotherapy with doxorubicin (total dose $375 \mathrm{mg} / \mathrm{m} 2$ ) combined 
with ifosfomide (total dose $54 \mathrm{mg} / \mathrm{m} 2$ ) and radiation therapy (total dose $55.8 \mathrm{~Gy}$ ) on the anterior mediastinum. However, reports on adjuvant treatment of liposarcoma are of mostly case reports and clinical trial conclusions, and no clear evidence can prove the exact effect of radiotherapy and chemotherapy in liposarcoma. Nassif et al. [11] also pointed out that, due to the lack of meaningful chemotherapy response in ALT/WDL, gene targets such as CDK4 (Cyclin-Dependent Kinases 4) and MDM2 amplicons have gained popularity in current research.

It has been reported that CDK4, MDM2 and p16(MTS/multiple tumor suppressor 1) immunohistochemical triplet protein can function as an auxiliary diagnostic tool [12, 13]. MDM2 gene is a proto oncogene, which inhibits the transcriptional activity of tumor suppressor gene p53(Tumor Suppressor Gene 53) by blocking the N-terminal transactivated domain. At the same time, its transcription process can also be activated by p53. Therefore, when p53 is stable, MDM2 transcription is also induce, resulting in higher MDM2 protein levels. In addition, MDM2 also acts as a ligase, targeting itself and p53, and is degraded by proteasome [14]. MDM2 and CDK4 have become clinically relevant diagnostic indicators and targets as the result of their existence found in about $90 \%$ of the amplified sequences of 2q12-15 displayed by ALT/WDL. RG 7112 is an MDM2 antagonist. Previous studies have shown that nongenotoxic nutlin and genotoxic drugs synergistically activate p53 function. In a small proof-of-principle study, RG 7112 combined with doxorubicin can enhance advanced soft tissue activation of p53 gene in patients with sarcoma. However, this combination therapy has a high incidence of grade three and four hematological toxicity, and $60 \%$ and $45 \%$ of patients have experienced acute neutropenia or thrombocytopenia [15-17]. In the future the joint use of nutlin and non-toxic drugs will have more potential for avoiding the myelosuppressive side effects of doxorubicin. The phosphorylation of CDK4 can inactivate the function of retinoblastoma (RB) protein and make the cell cycle from $\mathrm{G} 1$ phase to $S$ phase uninhibited. The natural cell cycle regulation can be restored by CDK4 inhibition, which can prevent cell proliferation out of control. Palbociclib (PD 0332991), an oral specific inhibitor of CDK4/6, has recently been approved for combination with tamoxifen in the treatment of breast cancer. It has shown inhibitory activity in WDL and dedifferentiated liposarcoma. However, being MDM2 antagonists alike, CDK4 inhibitors are only used in clinical trials for liposarcoma, and its development is limited due to the lack of reliable biomarkers, the development of drug resistance or adaptability, and potential adverse events [18-20].

\section{Conclusion}

In summary, this article presents a case of giant atypical lipomatous tumor of posterior mediastinum in adolescent treated by surgery, and a literature review of related studies. Specifically, it includes the summary for the diagnosis and treatment process of ALT/WDL, and the discussion for the future development of molecular inhibitors. We believe that no other ways is superior than more surgery is for the treatment of mediastinal liposarcoma, but it still the truth that the complete removement of tumor cannot be achieved by surgery. Therefore, the treatment of residual tumor tissue, especially the way to intervene the key targets of pathophysiology and development of liposarcoma, has emerged as a hot 
research topic. Molecular inhibitors will be an important method for the treatment of liposarcoma in the future.

\section{Abbreviations}

NCCN: National Comprehensive Cancer Network; WDL/ALT: well-differentiated liposarcoma/Atypical lipomatous tumor; CT: computed tomography; MR: magnetic resonance; CD34: Antigens,CD34; ki-67: ki67 Antigen; MDM2: Murine Double Minute2; CHOP: DDIT3/DNA Damage Inducible Transcript 3; CDK4: Cyclin-Dependent Kinases 4; p16: MTS/multiple tumor suppressor 1; p53: Tumor Suppressor Gene 53; FISH: Fluorescence In suit Hybridization.

\section{Declarations}

Acknowledgements

Not applicable.

Authors' contributions

(I) Administrative support: Shu-Guang Zhu; (II) Surgical

operation: Yang Zhang, Kai Chen, Qing-Rong Ma, Zhuang-Rong Huang, and Shu-Guang Zhu; (IV) Data collection and follow-up: All authors; (V) Manuscript writing: All authors; (VI) Final approval of manuscript: All authors.

Funding

Natural Science Foundation of Guangdong Province (CN) (2017A030313856).

Availability of data and materials

Please contact author for data requests.

Ethics approval and consent to participate

Not applicable.

Consent for publication

Not applicable.

Competing interests

The authors declare that they have no competing interests. 


\section{References}

1. Ortega P, Suster D, Falconieri G, Zambrano E, Moran CA, Morrison C, et al. Liposarcomas of the posterior mediastinum: clinicopathologic study of 18 cases. Mod Pathol. 2015;28:721-31.

2. Kuhnen C, Mentzel T, Fisseler-Eckhoff A, Debiec-Rychter M, Sciot R. Atypical lipomatous tumor in a 14-year-old patient: distinction from lipoblastoma using FISH analysis. Virchows Archiv: an international journal of pathology. 2002;441:299-302.

3. Burt M, Ihde J, Hajdu S, Smith J, Bains M, Downey R, et al. Primary sarcomas of the mediastinum: results of therapy. J Thorac Cardiovasc Surg. 1998;115:671-80.

4. Ohta Y, Murata T, Tamura M, Sato $\mathrm{H}$, Kurumaya $\mathrm{H}$, Katayanagi K. Surgical resection of recurrent bilateral mediastinal liposarcoma through the clamshell approach. Ann Thorac Surg. 2004;77:18379.

5. Yan PENG, Haiyan WENG, Hui TANG. Clinicopathological analysis of atypical lipomatous tumors/well-diferentiated liposarcoma. Modern Oncology. 2012;20:2401-4.

6. Fukuhara S, Dimitrova K, Geller C, Hoffman D, Ko W, Tranbaugh R. Progressive dyspnea in patient with large mediastinal mass. J Cardiothorac Surg. 2014;9:6.

7. Chen M, Yang J, Zhu L, Zhou C, Zhao H. Primary intrathoracic liposarcoma: a clinicopathologic study and prognostic analysis of 23 cases. J Cardiothorac Surg. 2014;9:119.

8. Derbel O, Heudel P, Cropet C, Meeus P, Vaz G, Biron P, et al. Survival impact of centralization and clinical guidelines for soft tissue sarcoma (A prospective and exhaustive population-based cohort). PloS one. 2017;12:e0158406.

9. Klimstra D, Moran C, Perino G, Koss M, Rosai J. Liposarcoma of the anterior mediastinum and thymus. A clinicopathologic study of 28 cases. Am J Surg Pathol. 1995;19:782-91.

10. Saeed M, Plett S, Kim GE, Daldrup-Link H, Courtier J. Radiological-pathological correlation of pleomorphic liposarcoma of the anterior mediastinum in a 17-year-old girl. Pediatric radiology. 2010;40(Suppl 1):68-70.

11. Nassif N, Tseng W, Borges C, Chen P, Eisenberg B. Recent advances in the management of liposarcoma. F1000Research. 2016;5:2907.

12. Altun E, Yüksel S, Kaygusuz G, Yıldız HY. Diagnostic importance of clinicopathologic features and p16, CD34, MDM2 expression in differential diagnosis of adipocytic tumors. Acta Orthop Traumatol Turc. 2020;54:59-65.

13. Thway K, Jones RL, Noujaim J, Zaidi S, Miah AB, Fisher C. Dedifferentiated Liposarcoma: Updates on Morphology, Genetics, and Therapeutic Strategies. Adv Anat Pathol. 2016;23:30-40.

14. Riddle N, Gonzalez R, Bridge J, Antonia S, Bui M. A CD117 and CD34 immunoreactive sarcoma masquerading as a gastrointestinal stromal tumor: diagnostic pitfalls of ancillary studies in sarcoma. Cancer control: journal of the Moffitt Cancer Center. 2011;18:152-9.

15. Roy S, Laroche-Clary A, Verbeke S, Derieppe M, Italiano A. MDM2 Antagonists Induce a Paradoxical Activation of Erk1/2 through a P53-Dependent Mechanism in Dedifferentiated Liposarcomas: 
Implications for Combinatorial Strategies. Cancers. 2020;12.

16. Italiano A, Toulmonde M, Cioffi A, Penel N, Isambert N, Bompas E, et al. Advanced welldifferentiated/dedifferentiated liposarcomas: role of chemotherapy and survival. Annals of oncology: official journal of the European Society for Medical Oncology. 2012;23:1601-7.

17. Coll-Mulet L, Iglesias-Serret D, Santidrián A, Cosialls A, de Frias M, Castaño E, et al. MDM2 antagonists activate p53 and synergize with genotoxic drugs in B-cell chronic lymphocytic leukemia cells. Blood. 2006;107:4109-14.

18. Dickson MA, Tap WD, Keohan ML, D'Angelo SP, Gounder MM, Antonescu CR, et al. Phase Il trial of the CDK4 inhibitor PD0332991 in patients with advanced CDK4-amplified well-differentiated or dedifferentiated liposarcoma. Journal of clinical oncology: official journal of the American Society of Clinical Oncology. 2013;31:2024-8.

19. Dickson MA, Schwartz GK, Keohan ML, D'Angelo SP, Gounder MM, Chi P, et al. Progression-Free Survival Among Patients With Well-Differentiated or Dedifferentiated Liposarcoma Treated With CDK4 Inhibitor Palbociclib: A Phase 2 Clinical Trial. JAMA oncology. 2016;2:937-40.

20. Vijayaraghavan S, Karakas C, Doostan I, Chen X, Bui T, Yi M, et al. CDK4/6 and autophagy inhibitors synergistically induce senescence in $\mathrm{Rb}$ positive cytoplasmic cyclin E negative cancers. Nature communications. 2017;8:15916.

\section{Figures}



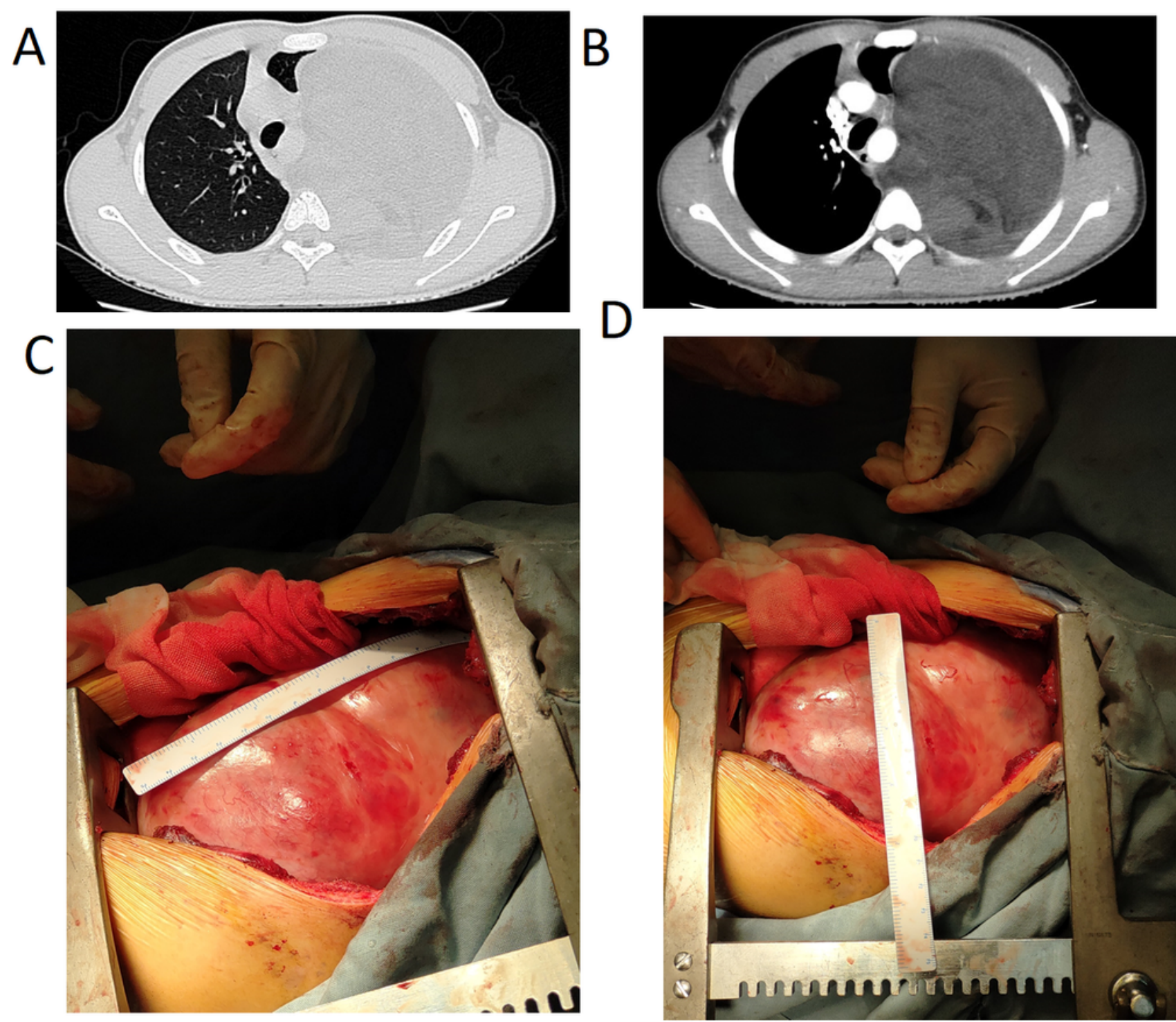

\section{$E$}

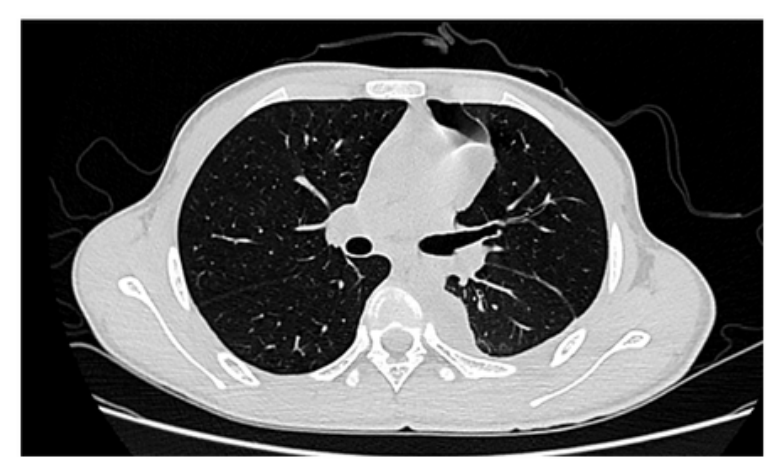

\section{Figure 1}

A: Left mediastinum-a huge mass be found in the left thoracic cavity and be compressing the adjacent left lung tissue. 1B: The enhancement shows uneven enhancement, which is considered to be swelling growth. 1C and 1D: The tumor seen during the operation is almost full of the chest cavity and is difficult to exposed. 1E: The recruitment of the left lung shows great. 

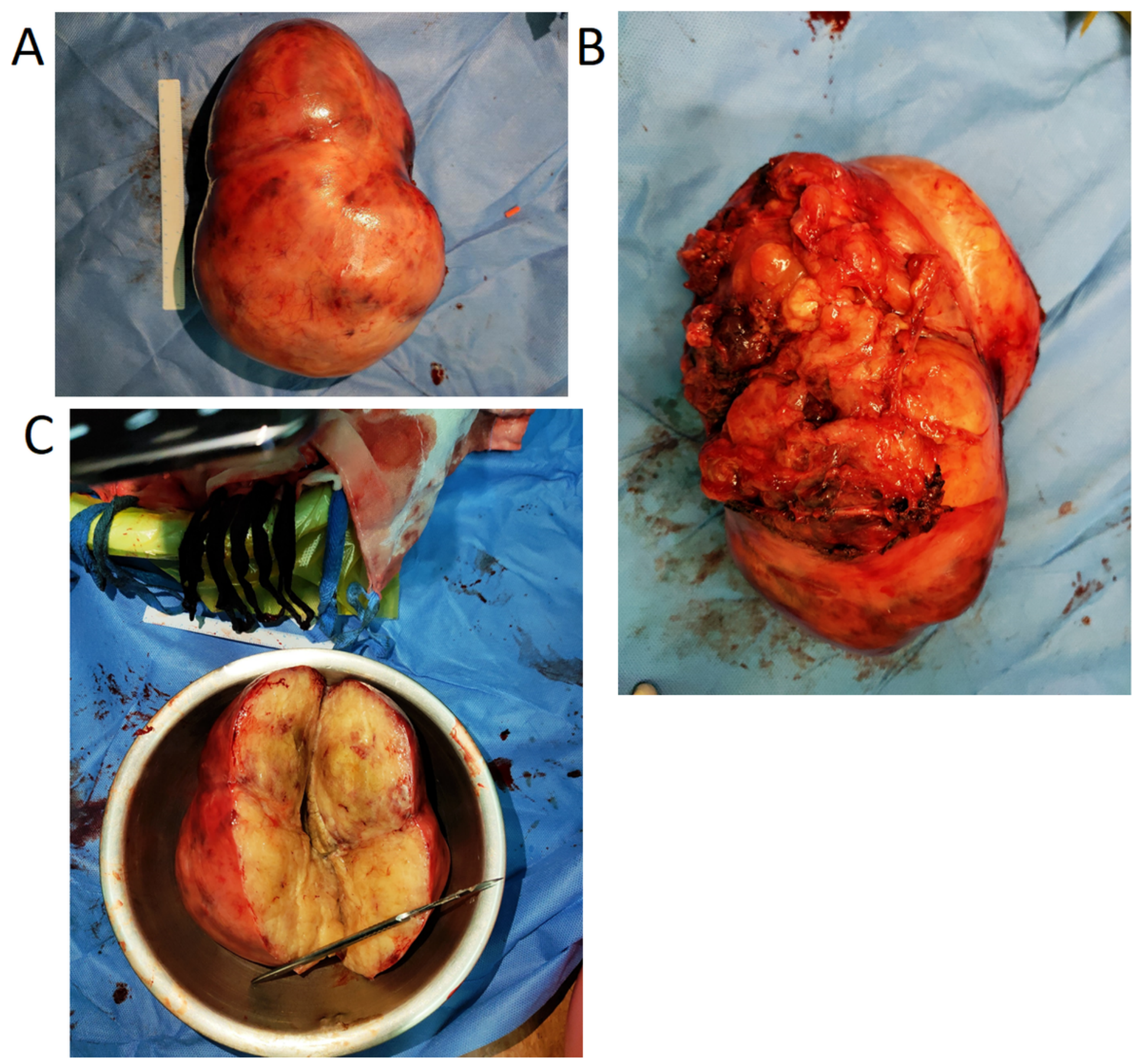

Figure 2

$\mathrm{A}$ is a front view of tumor, $2 \mathrm{~B}$ is an opposite view of the tumor and $2 \mathrm{C}$ is the appearance of the tissue in the cutting interface after tumor incision. 
A

B

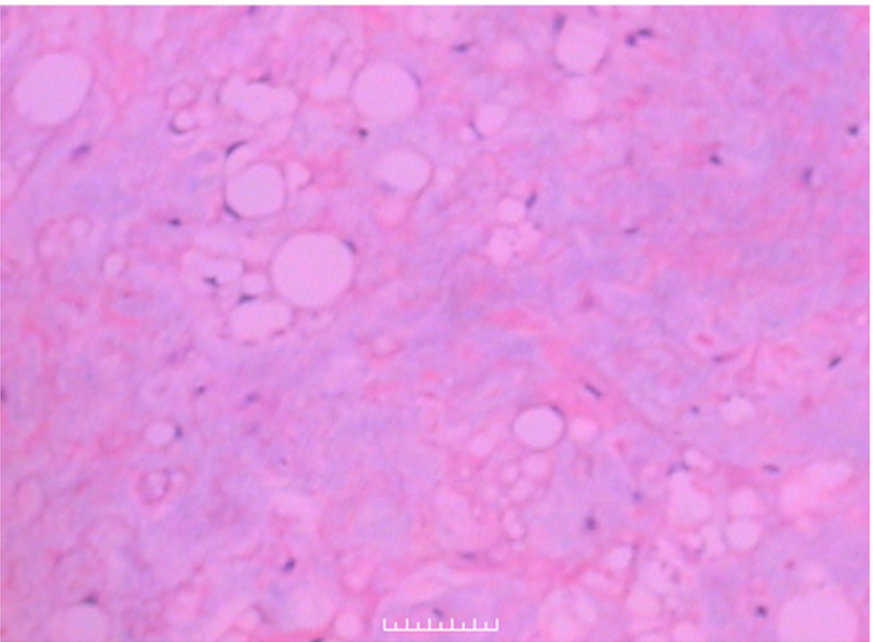

Figure 3

A: HE staining $\times 40$. B: HE staining $\times 100$. 